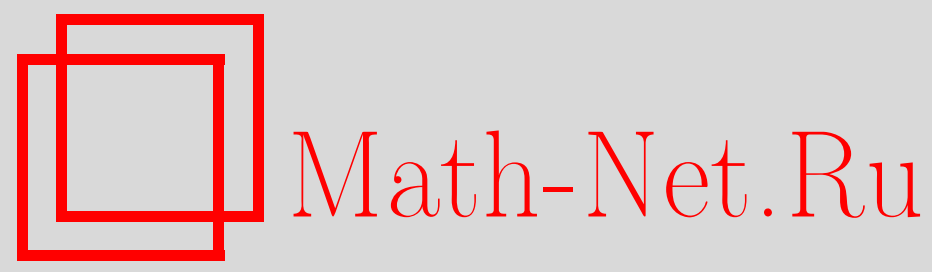

А. Ю. Зубов, О выборе оптимальной стратегии защиты для кода аутентификации с двумя состояниями источника, Дискрет. матем., 2009, том 21, выпуск 4, 135-147

DOI: https://doi.org/10.4213/dm1078

Использование Общероссийского математического портала Math-Net.Ru подразумевает, что вы прочитали и согласны с пользовательским соглашением http://www . mathnet.ru/rus/agreement

Параметры загрузки:

IP: 54.198 .187 .58

26 апреля 2023 г., 05:19:31 


\title{
О выборе оптимальной стратегии защиты для кода аутентификации с двумя состояниями источника
}

\author{
() 2009 г. А. Ю. Зубов
}

\begin{abstract}
Вычисляются точные значения вероятностей успеха активных атак для кода аутентификации с двумя состояниями источника.
\end{abstract}

Код аутентификации (далее $A$-код) - это математическая модель системы аутентификации информации. Как и шифр, код аутентификации имеет некоторое множество секретных ключей, которые используются для выбора правила кодирования. Как при шифровании, так и при аутентификации информации, выбор ключей осуществляется, как правило, случайно и равновероятно. Однако если такой выбор ключей положительно влияет на стойкость шифрования, то для кодов аутентификации это может быть и не так. Для некоторых конструкций $A$-кодов равновероятный выбор ключей может быть совсем неудачным. В данной статье приводится соответствующий пример.

Напомним основные определения и понятия. В литературе, посвященной кодам аутентификации (см., например, $[1,5,6])$, стороны, обменивающиеся информацией, называются передатчиком и приемником. Передатчик наблюдает состояния источника (например, результаты бросания монеты или битовые строки), с помощью некоторых правил кодирует их (используя секретные ключи), передавая получившиеся сообщения приемнику. Приемник восстанавливает передаваемое сообщением состояние источника и принимает полученное сообщение как аутентичное, используя установленный критерий аутентичности. Злоумышленник (называемый оппонентом) осуществляет активные атаки, к числу которых относятся атаки имитации и подмены. При попытке имитации оппонент вставляет, в канал связи поддельное сообщение, а при попытке подмены подменяет передаваемое сообщение поддельным сообщением. В обоих случаях оппонент рассчитывает на то, что поддельное сообщение будет принято как аутентичное. Не зная используемого передатчиком и приемником секретного ключа, оппонент может добиться успеха при выборе поддельного сообщения лишь с некоторой вероятностью. Если оппонент сможет определить по наблюдаемому сообщению секретный ключ, то он добьется успеха с вероятностью, равной 1. Чем меньше эта вероятность, тем более стойким является $A$-код. В простейшем случае речь идет об атаках на основе не более чем одного наблюдаемого сообщения. Более опасными могут быть атаки на основе ряда наблюдаемых сообщений (образованных, в частности, с помощью одного ключа). Мы ограничимся рассмотрением лишь простейших атак.

Формально $A$-код определяется следующим образом. Пусть $S, M, E$ - соответственно множества состояний источника, сообщений и правил кодирования. Каждое правило 
кодирования - это инъективное отображение $e: S \rightarrow M$. Оно определяет обратное отображение $e^{-1}: M \rightarrow S \cup\{0\}$ формулой

$$
e^{-1}(m)= \begin{cases}s \in S, & \text { если } m \in e(S), \text { причем } m=e(s), \\ 0, & \text { если } m \notin e(S),\end{cases}
$$

где

$$
e(S)=\{e(s): s \in S\}
$$

При этом полагают, что $0 \notin S$. Естественно, для любых $s \in S$ и $e \in E$ выполняется равенство

$$
e^{-1}(e(s))=s
$$

означающее, что сообщение и правило кодирования однозначно восстанавливают состояние источника. Обычно полагают, что выполняется равенство

$$
M=\bigcup_{e \in E} e(S)
$$

означающее, что каждое сообщение является результатом применения некоторого правила кодирования к некоторому состоянию источника. Код аутентификации - это тройка конечных множеств $(S, E, M)$, для которой выполняются условия (1) и (2). Для аутентификации передаваемых состояний источника передатчик и приемник выбирают (в секрете от оппонента) общее правило кодирования $e$. Передатчик вычисляет $m=e(s)$ и направляет сообщение $m$ приемнику. Критерием аутентичности полученного сообщения является условие $e^{-1}(m) \neq 0$. Для удобства выбора правил кодирования множество $E$ индексируется ключами.

Состояния источника появляются случайно, в соответствии с заданным на множестве $S$ распределением вероятностей

$$
\mathbf{P}(S)=\left(p_{S}(s), s \in S\right)
$$

Например, при случайном бросании монеты исходы (состояния источника) могут появляться как с равными, так и с разными вероятностями. Пусть $\widetilde{S}-$ случайная величина, определенная для любого $s \in S$ равенством

$$
\mathbf{P}\{\tilde{S}=s\}=p_{S}(s) .
$$

Передатчик и приемник выбирают правила кодирования из множества $E$ случайно в соответствии с некоторым распределением вероятностей

$$
P(E)=\left(p_{E}(e), e \in E\right) .
$$

Пусть $\widetilde{E}-$ соответствующая случайная величина.

По определению случайные величины $\widetilde{S}$ и $\widetilde{E}$ полагаются независимыми. Они индуцируют случайную величину $\widetilde{M}$ с множеством исходов $M$. Вероятность $p_{M}(m)$ вычисляется по формуле

$$
p_{M}(m)=\sum_{e \in E(m)} p_{E}(e) p_{S}\left(e^{-1}(m)\right),
$$


где $m \in M$,

$$
E(m)=\left\{e \in E: e^{-1}(m) \neq 0\right\} .
$$

$A$-код можно задать $|E| \times|M|$ матрицей, называемой матрицей кодирования, строки которой занумерованы правилами кодирования $e \in E$, столбцы - сообщениями $m \in M$; на пересечении строки матрицы с номером $e$ и столбца с номером $m$ расположен элемент $e^{-1}(m)$.

Следуя работам Г. Симмонса ([3-6]), будем оценивать стойкость $A$-кода к атаке имитации вероятностью $p_{0}$, определяемой следующим образом. Пусть $P(E)=P$ - произвольное распределение и $q-$ вероятность события $m \in e(S)$ при случайном выборе $e \in E$. Это - вероятность того, что сообщение $m \in M$ будет принято как аутентичное. Она выражается формулой

$$
p_{M}(m)=\sum_{e \in E(m)} p_{E}(e) .
$$

Вероятность $p_{0}$ определяется равенством

$$
p_{0}=\min _{P(E)} \max _{m} p_{M}(m)
$$

Стойкость $A$-кода к атаке подмены будем оценивать вероятностью $p_{1}$, определяемой следующим образом. Пусть $P(E)=P$ - произвольное распределение и $m, n \in M-$ различные сообщения. Пусть $p_{s}(n \mid m)$ - вероятность того, что для случайно выбранной пары $(e, s)$ оппонент добьется успеха при подмене наблюдаемого сообщения $m \in M$ сообщением $n \neq m$. Эта вероятность вычисляется по формуле

$$
p_{s}(n \mid m)=\frac{1}{p_{M}(m)} \sum_{e \in E(m, n)} p_{E}(e) p_{S}\left(e^{-1}(m)\right),
$$

где

$$
E(m, n)=E(m) \cap E(n) .
$$

Пусть

$$
p_{s}(m)=\max _{n \neq m} p_{s}(n \mid m) .
$$

Тогда по определению

$$
p_{1}=\min _{P(E)} \sum_{m \in M} p_{s}(m) p_{M}(m)
$$

Если ограничиться возможностью оппонента использовать одну из рассмотренных атак, то стойкость $A$-кода оценивается величиной $\max \left\{p_{0}, p_{1}\right\}$. Эта величина зависит от распределения $P(E)$, которое называется стратегией защиты. Для некоторых $A$-кодов с небольшими значениями параметров $|S|,|E|,|M|$ (см., например, [1-3]) можно точно вычислить вероятности $p_{0}$ и $p_{1}$, а также указать распределения $P(E)$, на которых достигаются минимумы в (4) и (6) (оптимальные стратегии защиты). Вычислим точные значения вероятностей успеха активных атак для $A$-кода с двумя состояниями источника и одинаковым числом правил кодирования и сообщений. 
Пусть $S=\{H, T\}$. Состояния источника $H$ и $T$ можно интерпретировать, например, как результаты случайного подбрасывания монеты (орел и решка), которые появляются с вероятностями $p$ и $1-p$ соответственно, $p \geqslant 0,5$. Пусть множества $E$ и $M$ состоят из $N$ элементов:

$$
E=\left\{e_{1}, e_{2}, \ldots, e_{N}\right\}, \quad M=\left\{m_{1}, m_{2}, \ldots, m_{N}\right\},
$$

где $N$ - произвольное натуральное число, $N \geqslant 3$. Пусть матрица кодирования (размера $N \times N)$ имеет вид

$$
\left(\begin{array}{cccccccc}
H & T & 0 & 0 & \ldots & 0 & 0 & 0 \\
0 & H & T & 0 & \ldots & 0 & 0 & 0 \\
\ldots \ldots & \ldots & \ldots \ldots \ldots \ldots & \ldots \ldots & \ldots \\
0 & 0 & 0 & 0 & \ldots & 0 & H & T \\
H & 0 & 0 & 0 & \ldots & 0 & 0 & T
\end{array}\right)
$$

Будем полагать, что $i$-я строка матрицы пронумерована правилом кодирования $e_{i}$, а $j$-й столбец - сообщением $m_{j}, i, j=1,2, \ldots, N$. Вычислим для данного $A$-кода вероятности $p_{0}$ и $p_{1}$, а также оптимальные стратегии защиты от имитации и от подмены, при $N=4$ это сделано в [2].

Пусть $p_{E}\left(e_{i}\right)=x_{i}, i=1,2, \ldots, N$. Тогда, согласно (4), $p_{0}$ совпадает с $\min _{\bar{x} \in \Omega} L(\bar{x})$, где

$$
\begin{aligned}
L(\bar{x}) & =\max \left\{x_{1}+x_{N}, x_{1}+x_{2}, x_{2}+x_{3}, \ldots, x_{N-1}+x_{N}\right\}, \\
\Omega & =\left\{\bar{x}=\left(x_{1}, \ldots, x_{N}\right): x_{1}+\ldots+x_{N}=1,0 \leqslant x_{i} \leqslant 1, i=1,2, \ldots, N\right\} .
\end{aligned}
$$

Нетрудно видеть, что минимальное значение $L(\bar{x})$ достигается лишь в случае, когда все переменные принимают одинаковые значения. Поэтому $p_{0}=2 / N$ и оптимальной стратегией защиты от имитации является равномерное распределение $P(E)$.

Рассмотрим атаку подмены. Согласно (6), $p_{1}$ совпадает с $\min \left\{L_{1}(\bar{x}), \bar{x} \in \Omega\right\}$, где

$$
\begin{array}{r}
L_{1}(\bar{x})=\max \left\{p x_{1} ; p x_{N}\right\}+\max \left\{(1-p) x_{1} ; p x_{2}\right\}+\max \left\{(1-p) x_{2} ; p x_{3}\right\}+\ldots \\
+\max \left\{(1-p) x_{N-2} ; p x_{N-1}\right\}+\max \left\{(1-p) x_{N-1} ;(1-p) x_{N}\right\} .
\end{array}
$$

Введем обозначение

$$
a=\frac{1-p}{p} .
$$

Теорема 1. Для рассматриваемого $A$-кода $p_{1}=0,5$, если $p=0,5$. Оптимальной стратегией защиты от подмены является равномерное распределение $P(E)$. При $p>0,5$ имеет место формула

$$
p_{1}=\frac{3 p-1-p^{2}\left(1+a^{N-1}\right)}{3 p-1-p a^{N-1}} .
$$

Оптимальная стратегия защчиты от подмены определяется вектором $\bar{x}$, где

$$
\begin{aligned}
x_{N} & =x_{1}=\frac{2 p-1}{3 p-1-p a^{N-1}}, \\
x_{2} & =\frac{a(2 p-1)}{3 p-1-p a^{N-1}}, \\
& \ldots \\
x_{N-1} & =\frac{a^{N-2}(2 p-1)}{3 p-1-p a^{N-1}} .
\end{aligned}
$$


Доказательство. При $p=0,5$ утверждение очевидным образом следует из вида функции $L_{1}(\bar{x})$. Пусть $p>0,5$. Будем вычислять

$$
p_{1}=\min \left\{L_{1}(\bar{x}), \bar{x} \in \Omega\right\}
$$

путем раскрытия максимумов в сумме правой части равенства (7) в $2^{N}$ случаях, определяемых расстановкой неравенств $\leqslant$ или $\geqslant$ вместо знака $\vee$ в системе

$$
\begin{aligned}
& p x_{1} \vee p x_{N}, \\
&(1-p) x_{1} \vee p x_{2}, \\
& \cdots \\
&(1-p) x_{N-2} \vee p x_{N-1}, \\
&(1-p) x_{N-1} \vee(1-p) x_{N} .
\end{aligned}
$$

В каждом таком случае $L_{1}(\bar{x})$ представляет собой линейную функцию с положительными коэффициентами. Представим для удобства систему (10) в виде

$$
\begin{aligned}
x_{1} & \vee x_{N}, \\
a x_{1} & \vee x_{2}, \\
\cdots & \\
a x_{N-2} & \vee x_{N-1}, \\
x_{N-1} & \vee x_{N}
\end{aligned}
$$

и расположим переменные $x_{1}, x_{N}, x_{N-1}, a x_{N-2}, a^{2} x_{N-3}, \ldots, a^{N-2} x_{1}$ в одну цепочку, которую для наглядности изобразим в виде схемы

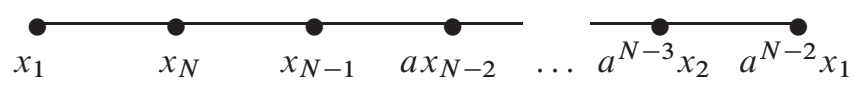

В схеме (12), соответствующей системе (11), на рисунках приведем стрелки, указывающие возрастание переменных:
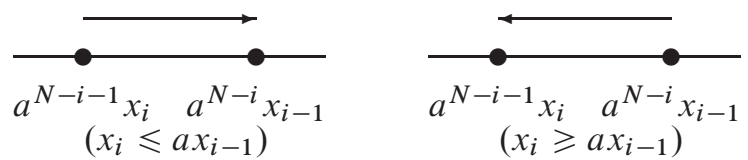

Заметим, что в схеме (12) могут быть точки трех видов, назовем их соответственно начальными, конечными и проходными. Начальная точка $a^{N-i-1} x_{i}$ отвечает случаю, когда в системе (11) имеются неравенства $a x_{i-1} \geqslant x_{i}$ и $a x_{i} \leqslant x_{i+1}$. Ей соответствует фрагмент схемы вида

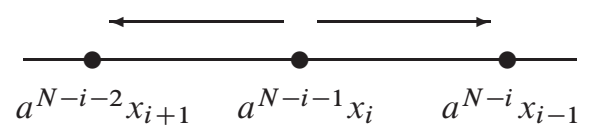

Конечная точка $a^{N-i-1} x_{i}$ отвечает случаю, когда в системе (11) имеются неравенства $a x_{i-1} \leqslant x_{i}$ и $a x_{i} \geqslant x_{i+1}$. Ей соответствует фрагмент схемы вида

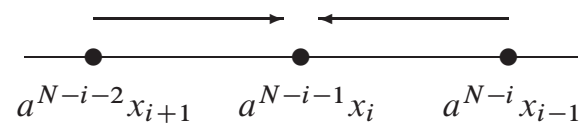


Наконец, проходная точка $a^{N-i-1} x_{i}$ отвечает случаю, когда в системе (11) имеются неравенства $a x_{i-1} \leqslant x_{i}$ и $a x_{i} \leqslant x_{i+1}$, или неравенства $a x_{i-1} \geqslant x_{i}$ и $a x_{i} \geqslant x_{i+1}$. Проходной точке соответствует один из фрагментов схемы вида

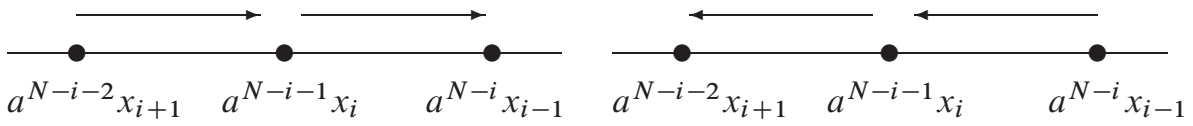

Нетрудно видеть, что в любом случае, определяемом системой (11), выражение функции $L_{1}(\bar{x})$ содержит переменную $x_{i}$ с коэффициентом 1 , если точка $a^{N-i-1} x_{i}$ конечная, с коэффициентом $p$ или $1-p$, если точка $a^{N-i-1} x_{i}$ проходная, и не содержит $x_{i}$, если точка $a^{N-i-1} x_{i}$ начальная.

Рассмотрим сначала два случая, в которых соответствующая схема не содержит начальных и конечных точек:

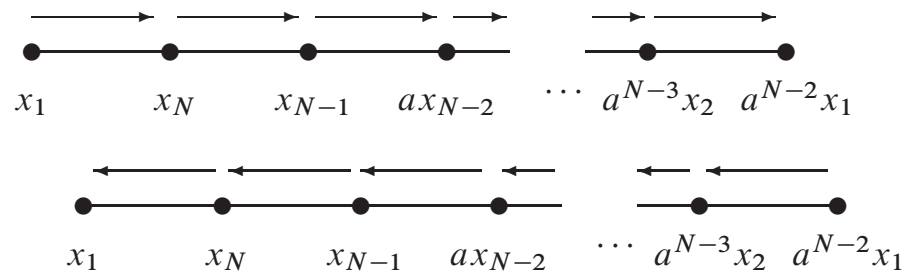

Первый случай невозможен, поскольку (в силу того, что $a<1$ ) его условия

$$
x_{1} \leqslant x_{N} \leqslant x_{N-1} \leqslant a x_{N-2} \leqslant \ldots \leqslant a^{N-3} x_{2} \leqslant a^{N-2} x_{1}
$$

противоречивы. Второй случай соответствует системе неравенств

$$
x_{1} \geqslant x_{N} \geqslant x_{N-1} \geqslant a x_{N-2} \geqslant \ldots \geqslant a^{N-3} x_{2} \geqslant a^{N-2} x_{1} .
$$

В этом случае $L_{1}(\bar{x})$ имеет вид

$$
\begin{aligned}
L_{1}(\bar{x}) & =p\left(x_{1}+x_{2}+\ldots+x_{N-1}\right)+(1-p) x_{N} \\
& =(2 p-1)\left(x_{1}+x_{2}+\ldots+x_{N-1}\right)+1-p .
\end{aligned}
$$

Лемма 1. В условиях (13) минимум функиии $L_{1}(\bar{x})$ достигается на наборе переменньх, удовлетворяющем условиям

$$
x_{N-1}=a x_{N-2}=\ldots=a^{N-3} x_{2}=a^{N-2} x_{1}=a^{N-2} x_{N} .
$$

При этом

$$
\min L_{1}(\bar{x})=\frac{3 p-1-p^{2}\left(1-a^{N-1}\right)}{3 p-1-p a^{N-1}},
$$

где минимум берется при выполнении условия (13).

Доказательство. Условия (13) можно представить в виде

$$
x_{N} \leqslant \frac{1}{a^{0}} x_{1} \leqslant \frac{1}{a^{1}} x_{2} \leqslant \ldots \leqslant \frac{1}{a^{N-2}} x_{N-1} .
$$


Пусть $\bar{x}^{1}-$ вектор аргументов, на котором $L_{1}(\bar{x})$ достигает минимума в условиях (17). Предположим, что в цепочке неравенств (17) имеет место хотя бы одно строгое неравенство:

$$
x_{N}^{1}=\frac{1}{a^{0}} x_{1}^{1}=\ldots=\frac{1}{a^{k-1}} x_{k}^{1}<\frac{1}{a^{k}} x_{k+1}^{1} \leqslant \ldots
$$

Рассмотрим тогда вектор $\bar{x}^{\prime}$ с координатами $x_{N}^{\prime}=x_{N}^{1}+\varepsilon, x_{1}^{\prime}=x_{1}^{1}+\varepsilon, \ldots, x_{k}^{\prime}=x_{k}^{1}+\varepsilon$, $x_{k+1}^{\prime}=x_{k+1}^{1}-(k+1) \varepsilon, x_{k+2}^{\prime}=x_{k+2}^{1}, \ldots, x_{N-1}^{\prime}=x_{N-1}^{1}$.

Очевидно, что $\varepsilon$ можно выбрать так, чтобы сумма координат $\bar{x}^{\prime}$ оставалась равной единице и выполнялись условия (17). Но

$$
L_{1}\left(\bar{x}^{\prime}\right)=L_{1}\left(\bar{x}^{1}\right)-(2 p-1) \varepsilon<L_{1}\left(\bar{x}^{1}\right) .
$$

Полученное противоречие доказывает, что координаты вектора $\bar{x}$, для которого $L_{1}(\bar{x})$ достигает минимума в рассматриваемых условиях, удовлетворяют условиям (15). Поскольку сумма координат вектора $\bar{x}$ равна 1 , получаем из (15) значения координат вектора $\bar{x}^{1}$. Они выражаются формулами (9). Учитывая, что в условиях леммы $L_{1}(\bar{x})$ можно выразить формулой $L_{1}(\bar{x})=p-(2 p-1) x_{N}$, из (9) получаем формулу (16).

Лемма 2. Пусть $\bar{x}^{(\alpha)}$ - вектор, для которого $\min L_{1}(\bar{x})=L_{1}\left(\bar{x}^{(\alpha)}\right)$ в условиях случая, определенного произвольной фиксированной совместной системой неравенств (11). Тогда координаты вектора $\bar{x}^{(\alpha)}$ удовлетворяют одному из следующих условий:

$$
\begin{aligned}
\alpha=0: \quad x_{N}^{(0)} & =x_{N-1}^{(0)}=a x_{N-2}^{(0)}=\ldots=a^{N-2} x_{1}^{(0)} ; \\
\alpha=1: \quad x_{N-1}^{(1)} & =a x_{N-2}^{(1)}=\ldots=a^{N-2} x_{1}^{(1)}=a^{N-2} x_{N}^{(1)} ; \\
\alpha=k: \quad x_{N-k}^{(k)} & =a x_{N-k-1}^{(k)}=\ldots=a^{N-k-1} x_{1}^{(k)}=a^{N-k-1} x_{N}^{(k)} \\
& =a^{N-k-1} x_{N-1}^{(k)}=a^{N-k} x_{N-2}^{(k)}=\ldots=a^{N-3} x_{N-k+1}^{(k)}, \quad 2 \leqslant k \leqslant N-1 .
\end{aligned}
$$

Доказательство. Случай, когда схема (12), соответствующая данной системе неравенств, не содержит начальных точек, рассмотрен в лемме 1 . При этом $\bar{x}^{(\alpha)}$ удовлетворяет условиям (15), которые совпадают с условиями случая $\alpha=1$. В других случаях схема имеет хотя бы одну начальную и хотя бы одну конечную точку. Рассмотрим фрагмент схемы, в котором начальная точка расположена между двумя конечными точками:


причем $m>1$. Такому фрагменту соответствует система неравенств

$$
\begin{aligned}
& a^{N-j-1} x_{j} \leqslant a^{N-j} x_{j-1} \leqslant \ldots \leqslant a^{N-k-1} x_{k}, \\
& a^{N-j-1} x_{j} \leqslant a^{N-j-2} x_{j+1} \leqslant \ldots \leqslant a^{N-m-1} x_{m} .
\end{aligned}
$$

Тогда точно так же, как в лемме 1 , можно доказать, что для координат вектора $\bar{x}^{(\alpha)}$ в системе (19) все нестрогие неравенства обязаны быть равенствами. Тем самым, выполняются равенства

$$
\frac{1}{a^{j-m}} x_{m}^{(\alpha)}=\ldots=\frac{1}{a} x_{j+1}^{(\alpha)}=x_{j}^{(\alpha)}=a x_{j-1}^{(\alpha)}=\ldots=a^{j-1} x_{k}^{(\alpha)} .
$$


Если для вектора $\bar{x}^{(\alpha)}$ выполняется условие (20), то будем говорить, что в схеме (12) на отрезке $\left[x_{m}^{(\alpha)}, x_{k}^{(\alpha)}\right]$ достигается равенство.

Пусть системе неравенств (11) соответствует схема (12), в которой имеется более одной начальной точки. Рассмотрим тогда фрагмент схемы, содержащий две соседние начальные точки $a^{N-j-1} x_{j}$ и $a^{N-s-1} x_{s}$ :

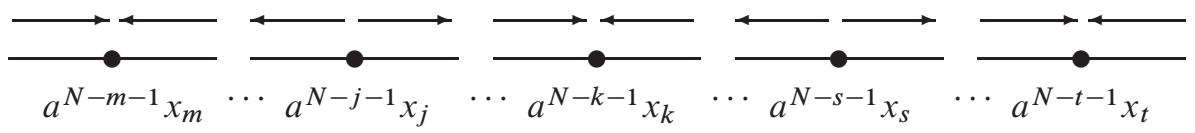

причем $m \neq 1$. Тогда для вектора $\bar{x}^{(\alpha)}$ достигаются равенства на отрезках $\left[x_{m}^{(\alpha)}, x_{k}^{(\alpha)}\right]$ и $\left[x_{k}^{(\alpha)}, x_{t}^{(\alpha)}\right]$. А так как эти отрезки пересекаются в точке $a^{N-k-1} x_{k}$, достигается равенство и на всем отрезке $\left[x_{m}^{(\alpha)}, x_{t}^{(\alpha)}\right]$. Таким образом, для нахождения вектора $\bar{x}^{(\alpha)}$, минимизирующего $L_{1}(\bar{x})$ в условиях (11), достаточно рассмотреть случай, когда соответствующая схема содержит ровно одну начальную точку. При этом и конечная точка должна быть одна, так как иначе в соответствующей схеме

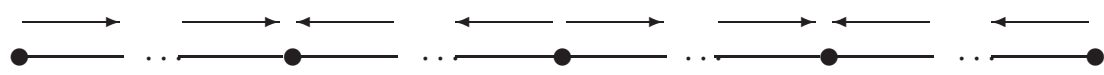

последняя точка, $a^{N-2} x_{1}$, является фактически второй начальной точкой.

Осталось рассмотреть возможные случаи расположения начальной и конечной точек в схеме, включая случай, когда конечная точка является первой или последней точкой схемы (при этом $m=1$ ).

Рассмотрим случай, когда конечная точка $a^{N-m-1} x_{m}$ не совпадает с последними двумя точками схемы $a^{N-3} x_{2}$ и $a^{N-2} x_{1}$ :

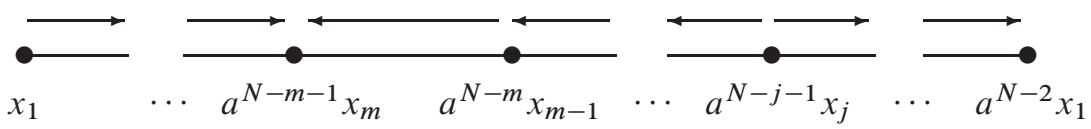

В этом случае имеет место система неравенств

$$
\begin{aligned}
\left(1 / a^{j-1}\right) x_{j} & \leqslant \ldots \leqslant(1 / a) x_{2} \leqslant x_{1} \leqslant x_{N} \leqslant \ldots \leqslant a^{N-m-1} x_{m}, \\
x_{j} \leqslant(1 / a) x_{j+1} & \leqslant \ldots \leqslant\left(1 / a^{m-j}\right) x_{m}
\end{aligned}
$$

Как и в доказательстве леммы 1 , легко убедиться в том, что необходимым для вектора $\bar{x}^{(\alpha)}$, минимизирующего $L_{1}(\bar{x})$, является условие, когда в неравенствах системы (21) достигаются равенства. Исключение составляют последние неравенства цепочек, которые можно записать в виде $a x_{m-1} \leqslant x_{m}$ и $x_{m+1} \leqslant a x_{m}$. Если бы выполнялись равенства в обоих этих неравенствах, мы получили бы противоречивое равенство $x_{1}=a^{N-2} x_{1}$. Отсюда следует, что для координат вектора $\bar{x}^{(\alpha)}$ справедливо одно из трех условий:

$$
\begin{aligned}
\left(1 / a^{m-2}\right) x_{m-1}^{(\alpha)}=\ldots & =\left(1 / a^{j}\right) x_{j+1}^{(\alpha)}=\left(1 / a^{j-1}\right) x_{j}^{(\alpha)}=\ldots \\
& =(1 / a) x_{2}^{(\alpha)}=x_{1}^{(\alpha)}=x_{N}^{(\alpha)}=\ldots=a^{N-m-1} x_{m}^{(\alpha)}, \\
\left(1 / a^{m-1}\right) x_{m}^{(\alpha)}=\ldots & =\left(1 / a^{j}\right) x_{j+1}^{(\alpha)}=\left(1 / a^{j-1}\right) x_{j}^{(\alpha)}=\ldots \\
& =(1 / a) x_{2}^{(\alpha)}=x_{1}^{(\alpha)}=x_{N}^{(\alpha)}=\ldots=a^{N-m-2} x_{m+1}^{(\alpha)}
\end{aligned}
$$


или

$$
\begin{aligned}
\left(1 / a^{m-2}\right) x_{m-1}^{(\alpha)}=\ldots & =\left(1 / a^{j}\right) x_{j+1}^{(\alpha)}=\ldots=(1 / a) x_{2}^{(\alpha)}=x_{1}^{(\alpha)}=x_{N}^{(\alpha)} \\
& =a^{N-m-2} x_{m+1}^{(\alpha)}<a^{N-m-1} x_{m}^{(\alpha)} .
\end{aligned}
$$

Их можно переписать соответственно в виде

$$
\begin{aligned}
x_{m-1}^{(\alpha)} & =a x_{m-2}^{(\alpha)}=\ldots=a^{m-3} x_{2}^{(\alpha)}=a^{m-2} x_{1}^{(\alpha)} \\
& =a^{m-2} x_{N}^{(\alpha)}=a^{m-2} x_{N-1}^{(\alpha)}=\ldots=a^{N-3} x_{m}^{(\alpha)}, \\
x_{m}^{(\alpha)} & =a x_{m-1}^{(\alpha)}=\ldots=a^{m-2} x_{2}^{(\alpha)}=a^{m-1} x_{1}^{(\alpha)} \\
& =a^{m-1} x_{N}^{(\alpha)}=a^{m-1} x_{N-1}^{(\alpha)}=\ldots=a^{N-3} x_{m+1}^{(\alpha)},
\end{aligned}
$$

И

$$
\begin{aligned}
x_{m-1}^{(\alpha)} & =a x_{m-2}^{(\alpha)}=\ldots=a^{m-3} x_{2}^{(\alpha)}=a^{m-2} x_{1}^{(\alpha)}=a^{m-2} x_{N}^{(\alpha)} \\
& =a^{m-2} x_{N-1}^{(\alpha)}=\ldots=a^{N-4} x_{m+1}^{(\alpha)}<a^{N-3} x_{m}^{(\alpha)} .
\end{aligned}
$$

На самом деле, последний случай, соответствующий тому, что в (21) последнее неравенство в каждой из двух цепочек строгое, невозможен. Дело в том, что в условиях (21) функция $L_{1}(\bar{x})$ имеет вид

$$
L_{1}(\bar{x})=p\left(x_{m}+\ldots+x_{j+1}+x_{N}\right)+(1-p)\left(x_{1}+\ldots+x_{j-1}+x_{m}+\ldots+x_{N-1}\right) .
$$

Поэтому действует тот же метод, приводящий к противоречию с условием выбора $\bar{x}^{(\alpha)}$, что и в лемме 1 . А именно, достаточно рассмотреть вектор $\bar{x}^{\prime}$, связанный с $\bar{x}^{(\alpha)}$ соотношениями $x_{s}^{\prime}=x_{s}^{(\alpha)}+\varepsilon$ при $s \neq m, x_{m}^{\prime}=x_{m}^{(\alpha)}-\varepsilon(N-1)$, выбрать $\varepsilon$ таким, чтобы выполнялись условия (22), и сравнить значения $L_{1}\left(\bar{x}^{(\alpha)}\right)$ и $L_{1}\left(\bar{x}^{\prime}\right)$, используя (23).

Каждый из двух оставшихся вариантов $\bar{x}^{(\alpha)}$ может быть получен путем разбиения множества точек схемы (12) на два подмножества, соответствующих двум цепочкам в (21), и последующим приравниванием значений точек в каждом подмножестве. Разбиение иллюстрируется схемой, на которой разорвано одно звено:

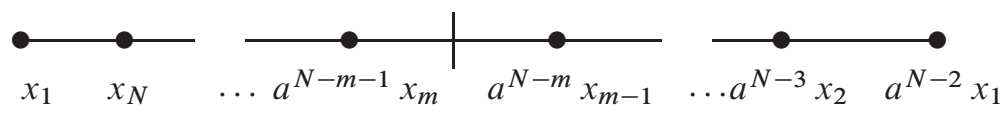

Таким разорванным звеном может быть любое из $N$ звеньев схемы. Непосредственно проверяется, что множество вариантов $\bar{x}^{(\alpha)}$ совпадает с множеством векторов, приведенных в формулировке леммы. Индекс $\alpha$ указывает номер (по счету слева) разрываемого звена схемы. При этом охватываются и случаи, когда конечной точкой схемы является одна из точек $a^{N-3} x_{2}$ или $a^{N-2} x_{1}$. Лемма доказана.

Непосредственно проверяется следующее утверждение.

Лемма 3. Вектор $\bar{x}^{(\alpha)}, 0 \leqslant \alpha \leqslant N-1$, определяемый условиями леммы 2 , имеет следу- 
ющчие координать:

$$
\begin{aligned}
x_{1}^{(0)} & =\frac{2 p-1}{p-(2-3 p) a^{N-2}}, \\
& \cdots \\
x_{N-2}^{(0)} & =\frac{a^{N-3}(2 p-1)}{p-(2-3 p) a^{N-2}}, \\
x_{N}^{(0)} & =x_{N-1}^{(0)}=\frac{a^{N-2}(2 p-1)}{p-(2-3 p) a^{N-2}},
\end{aligned}
$$

координаты $\bar{x}$ из (1) определяются формулами (9); при $2 \leqslant \alpha=k \leqslant N-1$

$$
\begin{aligned}
x_{1}^{(k)} & =x_{N}^{(k)}=x_{N-1}^{(k)}=\frac{a^{k-2}(2 p-1)}{p\left(1-a^{N-2}\right)+2(2 p-1) a^{k-2}}, \\
& \ldots \\
x_{N-k}^{(k)} & =\frac{a^{N-3}(2 p-1)}{p\left(1-a^{N-2}\right)+2(2 p-1) a^{k-2}}, \\
x_{N-k+1}^{(k)} & =\frac{(2 p-1)}{p\left(1-a^{N-2}\right)+2(2 p-1) a^{k-2}}, \\
& \ldots \\
x_{N-2}^{(k)} & =\frac{a^{k-3}(2 p-1)}{p\left(1-a^{N-2}\right)+2(2 p-1) a^{k-2}} .
\end{aligned}
$$

Для доказательства теоремы остается вычислить $L_{1}\left(\bar{x}^{(\alpha)}\right)$ для указанных вариантов векторов $\bar{x}^{(\alpha)}$ и найти среди полученных значений минимальное. Чтобы сделать это, заметим, что координаты всех $N$ векторов $\bar{x}^{(\alpha)}$ удовлетворяют системе неравенств (13). При этом

$$
L_{1}\left(\bar{x}^{(\alpha)}\right)=p-(2 p-1) x_{N}^{(\alpha)}
$$

Поэтому достаточно найти

$$
\max _{0 \leqslant \alpha \leqslant N-1} x_{N}^{(\alpha)}=x_{N}^{(l)},
$$

и вычислить $L_{1}\left(\bar{x}^{(l)}\right)$.

Используя лемму 3 , получаем, что

$$
\begin{aligned}
x_{N}^{(s)}-x_{N}^{(0)}=\frac{(2 p-1)\left[p-a^{N-2}\left(1-p a^{N-1}\right)\right]}{\left(3 p-1-p a^{N-1}\right)\left(p-(2-3 p) a^{N-2}\right)} & >0 \\
& \Longleftrightarrow p-a^{N-2}\left(1-p a^{N-1}\right)>0 .
\end{aligned}
$$

Пусть $b=a^{N-2}$. Тогда

$$
p-a^{N-2}\left(1-p a^{N-1}\right)=(1-p) b^{2}-b+p>0,
$$


поскольку $0<b<1$, и корнями квадратного трехчлена $(1-p) b^{2}-b+p$ являются $b_{1}=1$, $b_{2}=1 / a>1$. Аналогично,

$$
\begin{aligned}
x_{N}^{(1)}-x_{N}^{(k)}=\frac{(2 p-1)\left(p\left(1-a^{N-2}\right)+\right.}{\left.2(2 p-1) a^{k-2}-\left(3 p-1-p a^{N-1}\right) a^{k-2}\right)} & >0 \\
\left(3 p-1-p a^{N-1}\right)\left(p\left(1-a^{N-2}\right)+2(2 p-1) a^{k-2}\right) & \\
& \Longleftrightarrow p\left(1-a^{N-2}\right)-a^{k-2}\left(1-p-p a^{N-1}\right)>0 \\
& \Longleftrightarrow(1-b)\left(p-a^{k-2}(1-p)\right)>0 .
\end{aligned}
$$

Итак,

$$
\max _{0 \leqslant \alpha \leqslant N-1} x_{N}^{(\alpha)}=x_{N}^{(s)},
$$

и $L_{1}\left(\bar{x}^{(s)}\right)$ выражается формулой (16). Теорема доказана.

Наряду с введенными выше определениями вероятностей $p_{0}$ и $p_{1}$ используются и другие. Дело в том, что оценка этих вероятностей по формулам (4) и (6) в общем случае затруднительна. Это подтверждается тем, что (как показано в $[2,3]$ ) вычисление $p_{0}$ и $p_{1}$, а также соответствующих оптимальных стратегий защиты для $A$-кодов - это, по сути дела, решение матричных игр с матрицами весьма специфического вида. Хорошо известно, насколько сложны такие задачи. Значительно проще оцениваются вероятности успеха имитации и подмены в случае, когда используется единственная стратегия защиты - случайный и равновероятный выбор ключей $A$-кода. В этом случае вероятности $p_{0}$ и $p_{1}$ определяются формулами

$$
\begin{aligned}
\bar{p}_{0} & =\max _{m \in M} p_{M}(m)=\frac{1}{|E|} \max _{m \in M}|E(m)|, \\
\bar{p}_{1} & =\sum_{m \in M} p_{M}(m) p_{\mathrm{s}}(m) \\
& =\sum_{m \in M} \max _{n \neq m} \sum_{e \in E(m, n)} p_{E}(e) p_{S}\left(e^{-1}(m)\right) \\
& =\frac{1}{|E|} \sum_{m \in M} \max _{n \neq m} \sum_{e \in E(m, n)} p_{S}\left(e^{-1}(m)\right) .
\end{aligned}
$$

В частном случае, когда распределение $P(S)$ равномерно, формула (25) принимает вид

$$
\bar{p}_{1}=\frac{1}{|E||S|} \sum_{m \in M} \max _{n \neq m}|E(m, n)| .
$$

Определение вероятности успеха подмены по формулам (6) и (25) использует подход в среднем. Помимо этого, используется и подход в худшем случае, когда вероятность успеха подмены определяется как

$$
\begin{aligned}
p_{1}^{\prime} & =\min _{P(E)} \max _{m \in M} \max _{n \neq m} p_{S}(n \mid m) \\
& =\min _{P(E)} \max _{m \in M} \max _{n \neq m} \frac{1}{p_{M}(m)} \sum_{e \in E(m, n)} p_{E}(e) p_{S}\left(e^{-1}(m)\right),
\end{aligned}
$$


или (в случае, когда используется лишь равновероятный выбор ключей)

$$
\bar{p}_{1}^{\prime}=\max _{m \in M} \max _{n \neq m} \frac{\sum_{e \in E(m, n)} p_{S}\left(e^{-1}(m)\right)}{\sum_{e \in E(m)} p_{S}\left(e^{-1}(m)\right)} .
$$

В частном случае, когда и распределение $P(S)$ равномерно,

$$
\bar{p}_{1}^{\prime}=\max _{m \in M} \max _{n \neq m} \frac{|E(m, n)|}{|E(m)|} .
$$

Нетрудно видеть, что справедливы неравенства $p_{0} \leqslant \bar{p}_{0}, p_{1} \leqslant \bar{p}_{1}, p_{1} \leqslant p_{1}^{\prime}$ и $p_{1} \leqslant \bar{p}_{1}^{\prime}$. Поэтому, если для конкретной конструкции $A$-кода удалось получить оценку стойкости в виде неравенств $\bar{p}_{0}<\varepsilon$ и $\bar{p}_{1}<\varepsilon\left(p_{1}^{\prime}<\varepsilon, \bar{p}_{1}^{\prime}<\varepsilon\right)$, то будет иметь место и оценка стойкости в виде неравенств $p_{0}<\varepsilon$ и $p_{1}<\varepsilon$. Следовательно, любое предложенное определение вероятностей успеха имитации и подмены имеет право на существование. Однако величины $p_{1}, \bar{p}_{1}$ и $p_{1}^{\prime}, \bar{p}_{1}^{\prime}$ могут значительно различаться. Проиллюстрируем это на примере рассмотренного $A$-кода.

Согласно (7), равномерное распределение $P(E)$, используемое в качестве стратегии защиты от подмены для рассматриваемого $A$-кода, дает возможность оппоненту добиться успеха с вероятностью

$$
\begin{aligned}
\bar{p}_{1} & =\frac{1}{N}(p+(N-2) \max \{p ; 1-p\}+1-p) \\
& =\frac{1}{N}((N-2) p+1) .
\end{aligned}
$$

При $p \rightarrow 1$ эта величина стремится к $(N-1) / N$, в то время как значение $p_{1}$ стремится к 0,5 . Разность этих величин равна $(N-2) /(2 N)$. При достаточно больших значениях $N$ это практически 0,5 . Конечно, данный $A$-код нельзя считать стойким, поскольку для него $\max \left\{p_{0}, p_{1}\right\}>0,5$. Однако, если бы расхождение между вероятностями $p_{1}$ и $\bar{p}_{1}$ было таким же, как в рассмотренном примере, а величина $\max \left\{p_{0}, p_{1}\right\}$ не превосходила требуемого уровня стойкости $\varepsilon$, то такой $A$-код можно было бы признать стойким с позиции вероятностей $p_{0}$ и $p_{1}$, но нельзя признать стойким с позиции вероятностей $\bar{p}_{0}$ и $\bar{p}_{1}$. Тем самым, использование для оценки стойкости $A$-кода вероятностей $\bar{p}_{0}$ и $\bar{p}_{1}$ может, вообще говоря, оставить за рамками стойких и те $A$-коды, для которых выполняется неравенство $\max \left\{p_{0}, p_{1}\right\}<\varepsilon$.

Вычислим, наконец, для данного $A$-кода величины $p_{1}^{\prime}$ и $\bar{p}_{1}^{\prime}$. Согласно определению, для нахождения вероятности $p_{1}^{\prime}$ требуется вычислить минимум функции

$$
\begin{aligned}
& L_{1}^{\prime}(\bar{x})=\max \left\{\max \left(\frac{x_{1}}{x_{1}+x_{N}} ; \frac{x_{N}}{x_{1}+x_{N}}\right) ; \max \left(\frac{a x_{1}}{a x_{1}+x_{2}} ; \frac{x_{2}}{p x_{1}+p x_{2}}\right) ; \ldots ;\right. \\
& \left.\max \left(\frac{a x_{N-2}}{a x_{N-2}+x_{N-1}} ; \frac{x_{N-1}}{a x_{N-2}+x_{N-1}}\right) ; \max \left(\frac{x_{N-1}}{x_{N-1}+x_{N}} ; \frac{x_{N}}{x_{N-1}+x_{N}}\right)\right\} .
\end{aligned}
$$

Можно убедиться в том, что $\min _{\bar{x} \in \Omega} L_{1}^{\prime}(\bar{x})$ достигается на векторе

$$
\bar{x}=\left(\frac{1}{N}, \ldots, \frac{1}{N}\right)
$$


и указанный минимум равен $p$. Следовательно, $p_{1}^{\prime}=\bar{p}_{1}^{\prime}=p$.

Приведем таблицу точных значений вероятностей $p_{1}, \bar{p}_{1}, p_{1}^{\prime}, \bar{p}_{1}^{\prime}$ для рассмотренного примера $A$-кода при $p=0,6, p=0,9$ и $N=3,4,5,6$.

\begin{tabular}{|c|c|c|c|c|c|}
\hline \multirow{4}{*}{$p=0,6:$} & $N$ & 3 & 4 & 5 & 6 \\
\hline & $p_{1}$ & 0,525 & 0,533 & 0,541 & 0,545 \\
\hline & $\bar{p}_{1}^{\prime}$ & 0,533 & 0,550 & 0,560 & 0,566 \\
\hline & $\bar{p}_{1}^{\prime}=p_{1}^{\prime}$ & 0,600 & 0,600 & 0,600 & 0,600 \\
\hline \multirow{4}{*}{$p=0,9$} & $N$ & 3 & 4 & 5 & 6 \\
\hline & $p_{1}$ & 0,521 & 0,522 & 0,523 & 0,524 \\
\hline & $\bar{p}_{1}^{\prime}$ & 0,633 & 0,700 & 0,740 & 0,766 \\
\hline & $\bar{p}_{1}^{\prime}=p_{1}^{\prime}$ & 0,900 & 0,900 & 0,900 & 0,900 \\
\hline
\end{tabular}

\section{Список литературы}

1. Зубов А. Ю., Математика кодов аутентификации. Гелиос АРВ, Москва, 2007.

2. Зубов А. Ю., К теоретико-игровому подходу исследования кодов аутентификации. Дискретная математика (2009) 21, №3, 45-72.

3. G. J. Simmons, A game theoretical model of digital message authentication. Congressus Numerantium (1982) 34, 413-424.

4. Simmons G. J., Authentication theory/Coding theory. Lect. Notes Comput. Sci. (1985) 196, 411-432.

5. Симмонс Г. Дж., Обзор методов аутентификации информации. ТИИЭР (1988) 76, №5, 105-125.

6. Simmons G. J., A survey of information authentication. In: Contemporary Cryptology: The Science of Information Integrity (Simmons G. J., ed.). IEEE Press, New York, 1992, pp. 379-419.

Статья поступила 13.02.2008. 